\title{
Visualisation and interaction for scientific exploration and knowledge discovery
}

\author{
Elena Zudilova-Seinstra • Tony Adriaansen
}

Published online: 8 August 2007

(C) Springer-Verlag London Limited 2007

With the rapid development of information technology, the amount and complexity of scientific data increase exponentially every day. The data explosion has led to very large detailed datasets and the amount of detail in these datasets continues to grow. Nowadays, the challenge is to harness the power of knowledge hidden in the collage of scientific data.

Visualisation technologies empower users to perceive important patterns in the data, identify areas that need further scrutiny and make sophisticated decisions. However, looking at information is only the first step in understanding. For some domains, it is unclear what the features of interest are and how to define them in such a way that they can be detected. Many scientific discovery tasks can only be solved in interactive ways: by combining information analysis tools, advanced visualisation techniques and intuitive user interfaces. The importance of human interaction and perception cannot be ignored in the field of scientific computing and data analysis.

Interactive exploration systems allow people to examine scientific data and share obtained knowledge with, and through, information technology. These systems should be easy to use and not require sophisticated computer skills, as most of users are rather domain-specific experts than computer scientists. To increase users' abilities to explore the data and better understand the results of experiments based on extensive calculations, new scientific methods need to be developed and applied.

This special issue combines papers that discuss how modern visualisation and interaction techniques can augment the information analysis cycle from the user's perspective. The collected manuscripts are updated and expanded versions of papers presented at a series of

E. Zudilova-Seinstra ( $\square)$

Scientific Visualisation and Virtual Reality, Section Computational Science,

Faculty of Science, University of Amsterdam, Kruislaan 403,

Amsterdam 1098SJ, The Netherlands

e-mail: elenaz@science.uva.nl

T. Adriaansen

Commonwealth Scientific and Industrial Research Organisation, ICT Centre,

P.O. Box 76, Epping, NSW 2121, Australia

e-mail: tony.adriaansen@csiro.au 
international events related to topics of the interactive exploration and analysis of scientific data.

The issue opens with a paper by Cai et al. which presents the human-computer visual interaction approach to the data mining of complex spatio-temporal phenomena. In this project, multiple visualisations and human-computer interaction techniques are tightly coupled and embedded in the simulated dynamic process. The case study of this research is the simulation of the algae formation based on the Bayseian Cellular Automata model.

The second paper by Corrie and Storey is devoted to distributed scientific collaboration. Today's scientific problems require a wide range of expertise. As a result, collaborative exploration and visualisation environments are becoming increasingly important tools for the scientific research community. They allow opinions to be shared, removing the data bottleneck of individual analysis and reducing the time to discovery. The paper explores the importance of gestures in distributed scientific collaboration, when remote collaborators work together using complex digital artefacts. Authors report results of an observational study and present quantitative analysis of gesture-based interactions during co-located and distributed scientific collaborations.

The third paper by Sas and Schmidt discusses a method for analysing trajectories followed by people while they perform navigational tasks in virtual environments. The maturing virtual reality (VR) techniques provide ground for new enabling tools addressing computer-supported activities. Quantitative analysis of user trajectories in a VR environment is an important topic in human factors studies, virtual reality technologies, medical evaluations and training. The spatial knowledge acquisition is of specific interest for this research, where the Bezier curve approximation has been chosen for the diagnosis of navigational patterns.

The next two papers of this issue deal with multi-modal interaction applied to the visualisation and exploration of scientific data.

The fourth paper by Kok and van Liere addresses an important topic of designing multimodal user interfaces. Multi-modal user interfaces facilitate richer interaction styles for both information retrieval and analysis. Based on the combination of several interaction modalities (i.e., visual display, speech, gestures, direct manipulation, haptics, real time video and audio, etc.), multi-modal interfaces enable people's interaction with the visual representations of phenomena and results of computer-based scientific experiments in a more intuitive and flexible way. The paper reports on novel methods for the 3D manipulation and depth perception in immersive VR, which enhance users' spatial interaction with the visualised scientific data. A 3D interface extension to the Kitware Visualisation Toolkit is presented.

The fifth paper by Evreinova et al. introduces a novel approach to non-visual user interactions supported by direction predictive sounds and kinesthetic feedback. The non-visual interface techniques allow users' interactions, which might be hard to achieve through the visual medium only. Audio and tactile feedback are the most important output modalities for visually impaired users. The paper presents two case studies suggesting that sonification can be used to support people to successfully inspect various graphical shapes in the absence of visual stimuli.

The last paper of this issue by Zudilova-Seinstra explores the importance of individual human abilities in the design of adaptive user interfaces for scientific problem solving environments. Adaptive user interfaces allow an interactive exploration environment to automatically learn and adapt to important user, task and environmental parameters. The paper reports results of an experimental study suggesting criteria for the inclusion of investigated human factors into the user model of an adaptive interface.

To conclude this editorial, we would like to thank all contributors for their work and people who made this special issue possible. We acknowledge the program committee members and 
external reviewers for their comments on manuscripts. Special thanks go to Judith KleinSeetharaman, Kamran Sedig, Corina Sas, Pilar Herrero, Yang Cai, Ross Brown, Noureddine Elouazizi, Alex Olwal, Xiaobai Yao, Judith Devaney, Vanessa Evers, Michael Scarpa and Nicu Sebe. Also, we are grateful to Caroline Dickerson from Springer, UK, for her help and support during the preparation of this special issue.

\section{Authors Biography}

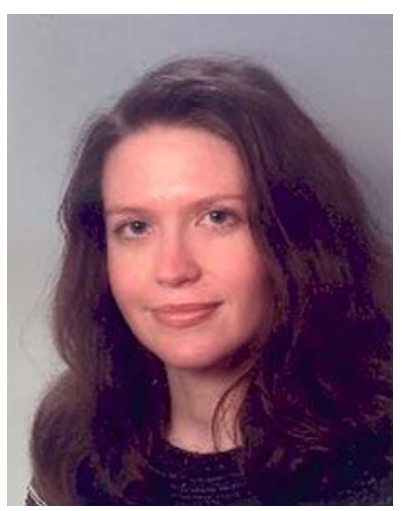

Elena Zudilova-Seinstra is a senior researcher at the Scientific Visualisation and Virtual Reality group of the University of Amsterdam. Previously she worked at the Corning Scientific Centre. Apart from being a researcher, in 1999-2002 she was a part-time assistant professor at the St. Petersburg Academy of Management Methods and Techniques. She received her MS degree in Technical Engineering in 1993 and PhD in Computer Science in 1998 from the St. Petersburg State Technical University. In 1996 she received an award for R\&D from the Welles-Johnson Foundation of Maryland. She is a program committee member of several international conferences and workshops. Her current research interests include multi-modal and adaptive interaction, scientific visualisation, virtual and augmented reality, ambient intelligence and usability studies. She has more than 40 research publications and three editorials in the above areas. Also, she has been an INTAS evaluator since February 2005.

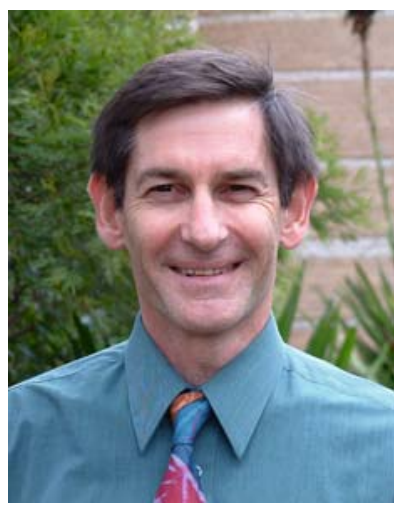

Tony Adriaansen is an experimental scientist at CSIRO, ICT Centre in Marsfield, Australia. He received a Bachelor degree in the School of Maths and Physics at Macquarie University and postgraduate diploma in electrical engineering and computing science from the University of NSW. His main work is in the "e-Health" group furthering research and modelling on the Networked Haptic Workbench, a virtual reality system with touch feedback. His research interests cover image analysis and pattern recognition, visualisation, VR and $3 \mathrm{D}$ environments, haptics, surgical simulation, perceptual colour theory, human vision and remote sensing. He is the treasurer of the National Australian Pattern Recognition Society, past Vice-President and is a member of the IEEE. He has been involved in research areas as diverse as analysis of LANDSAT and other satellite data, tropospheric aerosol trends, visualization of CSIRO's stratospheric ozone model, automatic detection and classification of wool staples and 3D modelling for virtual reality. 